Published in final edited form as:

J Am Chem Soc. 2019 September 18; 141(37): 14699-14706. doi:10.1021/jacs.9b06237.

\title{
Single-Atom Fluorescence Switch: A General Approach toward Visible-Light-Activated Dyes for Biological Imaging
}

\author{
Juan Tang ${ }^{\dagger, \# \text {, Michael A. Robichaux }}{ }^{\ddagger, \#}$, Kuan-Lin $\mathbf{W u}^{\dagger}$, Jingqi Peil ${ }^{\|}$, Nhung T. Nguyen $§$, Yubin \\ Zhou§, Theodore G. Wensel ${ }^{\star}, \ddagger$, Han Xiao ${ }^{\star}, \dagger, \|, \perp$ \\ tDepartment of Chemistry, Rice University, Houston, Texas, 77005, United States \\ "Department of Biosciences, Rice University, Houston, Texas, 77005, United States \\ ${ }^{\perp}$ Department of Bioengineering, Rice University, Houston, Texas, 77005, United States \\ ‡Verna and Marrs McLean Department of Biochemistry and Molecular Biology, Baylor College of \\ Medicine, Houston, Texas 77030, United States \\ $\S$ Center for Translational Cancer Research, Institute of Biosciences and Technology, College of \\ Medicine, Texas A\&M University, Houston, Texas 77030, United States
}

\begin{abstract}
Photoactivatable fluorophores afford powerful molecular tools to improve the spatial and temporal resolution of subcellular structures and dynamics. By performing a single sulfur-for-oxygen atom replacement within common fluorophores, we have developed a facile and general strategy to obtain photoactivatable fluorogenic dyes across a broad spectral range. Thiocarbonyl substitution within fluorophores results in significant loss of fluorescence via a photoinduced electron transferquenching mechanism as suggested by theoretical calculations. Significantly, upon exposure to air and visible light residing in their absorption regime (365-630 $\mathrm{nm}$ ), thio-caged fluorophores can be efficiently desulfurized to their oxo derivatives, thus restoring strong emission of the fluorophores. The effective photoactivation makes thio-caged fluorophores promising candidates for superresolution imaging, which was realized by photoactivated localization microscopy (PALM) with low-power activation light under physiological conditions in the absence of cytotoxic additives (e.g., thiols, oxygen scavengers), a feature superior to traditional PALM probes. The versatility of this thio-caging strategy was further demonstrated by multicolor super-resolution imaging of lipid droplets and proteins of interest.
\end{abstract}

\footnotetext{
*Corresponding Authors: han.xiao@ rice.edu. twensel@bcm.edu.

\#J.T. and M.R. contributed equally.

The authors declare no competing financial interest.

Supporting Information

The Supporting Information is available free of charge on the ACS Publications Web site., videos about the photoactivation and photobleaching. The Supporting Information is available free of charge on the ACS Publications website at DOI: 10.1021/jacs. $9 \mathrm{~b} 06237$.

Video 1: photoactivation of SNile Red in adipocytes (MOV)

Video 2: photobleaching of SNile Red in adipocytes (MOV)

Video 3: photoactivation of SDMAP-Halo in CHO-K1 cells (MOV)

Materials and instruments, experimental procedures, details concerning synthesis, NMR, MS, and IR characterization data, supplemental figures including additional theoretical calculations, photoactivation, stability, and cell imaging data (PDF)
} 


\section{Graphical Abstract}

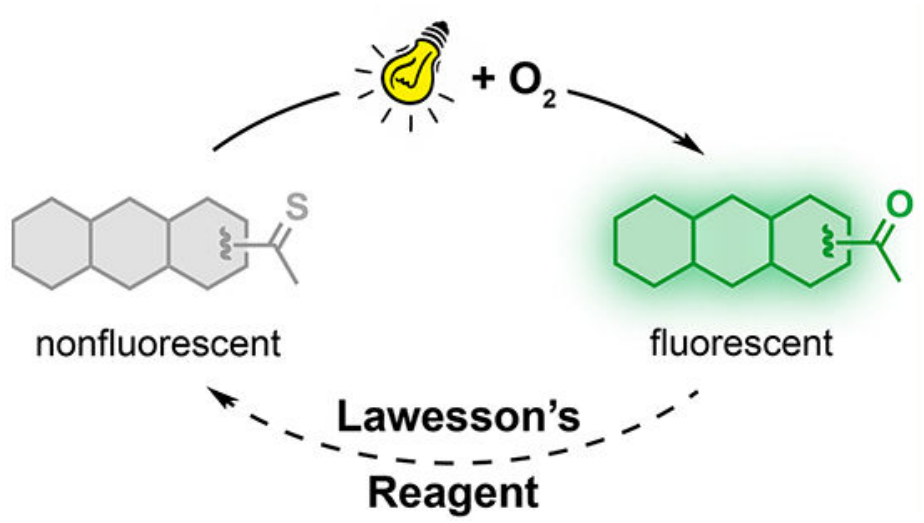

\section{INTRODUCTION}

Photoactivatable fluorophores, also called photocaged fluorophores, are an important class of optical probes for biological imaging. ${ }^{1-9}$ Upon irradiation with light of appropriate wavelengths, these photocaged fluorophores can undergo photochemical reactions that release the caged groups to regenerate fluorophores in their active forms. Because of their ability to turn on the target of interest with high spatial and temporal resolution in complex biological systems, photo-activatable fluorophores afford powerful tools for high resolution tracking of biological processes in living cells, tissues, and animals. Recently, sequential imaging of photo-activatable fluorophore-labeled molecules has enabled super-resolution imaging beyond the diffraction limit (e.g., Photo-activated Localization Microscopy, PALM; Stochastic Optical Reconstruction Microscopy, STORM), revealing previously unobserved details of biological structures and processes. ${ }^{10,11}$

To develop photoactivatable probes for biological studies, photocleavable "cage" functional groups, including $o$-nitrobenzyl, phenacyl, and azidophenyl moieties, have been conjugated to fluorophores. ${ }^{3,4,11,12}$ In general, the resulting photoactivatable fluorophores have relatively large size and can only be efficiently cleaved by irradiation with ultraviolet light, dramatically limiting their applications in biological systems. To avoid the use of UV light, researchers have explored the use of indirectly visible and near-infrared light absorbing approaches, such as metal-ligand photocaging and photon up-conversion systems. ${ }^{12-15}$ However, these technologies require the use of a light-capturing sensitizer ${ }^{12,13}$ or an expensive multiphoton light source. ${ }^{14,15}$ Recently, computational approaches have been used to develop a new family of quenching BODIPY dyes that can be decaged by exposure to green light, in which meso-substituted BODIPY fluorophores release acetic acid to restore fluorescence. ${ }^{16-18}$ Unfortunately, this photoactivation mechanism utilizes the low excitation states of meso-substituted BODIPY analogues, a strategy that may not be easily applied to the design of other fluorophores. Therefore, our goal is to devise a general strategy for developing minimally modified visible-light-photoactivatable probes across a broad spectral range. 
To this end, we hypothesized that a single atom substitution of fluorophores, sulfur-foroxygen replacement, could be a general mechanism to quench fluorophores via a photoinduced electron transfer (PET) quenching mechanism (Figure 1). Recently, the thiocarbonyl group was reported to be an effective fluorescence quencher for nearby fluorophores. ${ }^{19-22}$ Thioamides have been chemically incorporated into proteins as fluorescence quenchers to facilitate the study of protein dynamics, folding, and aggregation. 19,20 This PET-quenching mechanism depends on the ability of the thioamide group to serve as an electron donor for the first excited singlet state of the fluorophore acceptor. ${ }^{21,22}$

Taking advantage of the PET-quenching mechanism, thiocoumarin probes have been developed for the detection of specific chemical species. In the presence of $\mathrm{Au}^{3+}, \mathrm{Hg}^{2+}$, or strong oxidizing agents, thiocoumarin derivatives with weak fluorescence signals can undergo oxidative desulfurization to their fluorescent oxo analogues. ${ }^{23-25}$ Because of the relatively large size of the sulfur atom, the orbital overlap of thiocarbonyl group is not as efficient as that of the carbonyl group, thus leading to higher energy highest occupied molecular orbitals (HOMOs) and lower energy lowest unoccupied molecular orbitals (LUMOs). The changes of frontier molecular orbitals make it possible to induce a PET effect within thio-caged fluorophores, and consequently quench the fluorescence. On the other hand, these thio-caged fluorophores can be photochemically oxidized to their native oxo forms, thus restoring their fluorescence. In the presence of UV light and oxygen, thioketones were reported to afford the corresponding carbonyl compounds. ${ }^{26,27} \mathrm{We}$ envision that conjugating the thiocarbonyl group to a large conjugated system, such as fluorophores, may enable the oxidization of these thiocarbonyl groups using light in the visible region (Figure 1).

Here, we report a series of fluorophores bearing one or two thiocarbonyl groups that could cover a broad spectral window. We found that introduction of a thiocarbonyl moiety into fluorophores leads to PET-induced fluorescence quenching, which can be reversed through oxidative desulfurization by exposure to air and visible light residing in their absorption regime $(365-630 \mathrm{~nm})$. As examples for applications, we have demonstrated the utility of these thio-caged fluorophores for multicolor super-resolution imaging by obtaining ultrastructural information on lipid droplets and PALM imaging of proteins of interest in combination with genetically encoded tagging technology. In contrast to traditional PALM or STORM probes which need high-power excitation with cytotoxic additives, such as thiols or oxygen scavengers, the thio-caged dyes hold great promise for living cell super-resolution imaging with low-power activation light in physiological conditions with no need of any imaging additives.

\section{RESULTS AND DISCUSSION}

\section{Synthesis and Characterization of Thio-Caged Nile Red.}

To determine whether a minimal one atom sulfur-for-oxygen substitution within fluorophores can lead to fluorescence quenching, we first synthesized thio-caged Nile Red (SNile Red, Figure 1), in which a carbonyl group was replaced with a thiocarbonyl moiety. Treatment of Nile Red with Lawesson's reagent in refluxing toluene produced the desired SNile Red in a yield of $40 \%$. SNile Red in DMSO solution exhibited a strong absorption 
band at $652 \mathrm{~nm}$, accompanied by weak fluorescence (Figure 2A). The fluorescence quantum yield of SNile Red $\left(\Phi_{\mathrm{f}}<0.001\right)$ in DMSO is significantly lower than that of the Nile Red $\left(\Phi_{\mathrm{f}}=0.46\right)$, indicating the likelihood that the oxidizing desulfuration reaction could be used to reactivate the fluorescence of SNile Red (Table 1). To our delight, irradiation of SNile Red with red light $\left(615 / 30 \mathrm{~nm}, 0.4 \mu \mathrm{W} \mathrm{cm}{ }^{-2}\right)$ led to the regeneration of fluorescent Nile Red in a high yield (Figure 2A). The absorption peaks of SNile Red at 298, 368, and $652 \mathrm{~nm}$ were blue-shifted after desulfuration to 315 and $556 \mathrm{~nm}$, in good agreement with the absorption spectrum of the commercially available Nile Red (Figure 2B). Upon irradiation with red light, the fluorescence intensity of the desulfurated molecule at $626 \mathrm{~nm}$ increased by 280 -fold (Figure 2C).

To evaluate the proper light source for SNile Red photoactivation, SNile Red was irradiated with $470 \mathrm{~nm}$ (470/60), $615 \mathrm{~nm}$ (615/30), and $850 \mathrm{~nm}$ (850/100) light, respectively, and the fluorescence intensities at $626 \mathrm{~nm}$ were recorded. As shown in Figure 2D, a significant fluorescence enhancement was observed by irradiation at 470 and $615 \mathrm{~nm}$. On the other hand, treatment of light out of the absorbance range of SNile Red $(850 \mathrm{~nm})$ induced negligible activation of SNile Red after $5 \mathrm{~h}$. These results demonstrated thio-caged fluorophores could be photoactivated using a visible-light source within the absorbance range of thio-caged dye rather than the UV light required in previous photoactivation protocols, ${ }^{3}$ a result which holds great promise for biological applications.

To further characterize the photoactivated product of SNile Red, ${ }^{1} \mathrm{H}$ NMR and ESI-MS spectral changes were measured in the process of photoactivation (Figure 2A and S1). Upon irradiation with red light $\left(615 / 30 \mathrm{~nm}, 0.4 \mu \mathrm{W} \mathrm{cm}{ }^{-2}\right)$, the aromatic protons exhibited an upfield shift. Signals corresponding to phenyl moiety protons at 8.64, 8.62, 7.85, 7.73, 7.36, 7.04 , and $6.79 \mathrm{ppm}$ gradually disappeared, and were replaced by new signals appearing at $8.57,8.13,7.81,7.72,7.63,6.85,6.69$, and $6.30 \mathrm{ppm}$, respectively. After $5 \mathrm{~h}$ of irradiation, the ${ }^{1} \mathrm{H}$ NMR spectrum of the final photoactivated product exhibited good agreement with that of Nile Red. The photoactivated product was also characterized by ESI-mass spectrometric analysis (Figure S1). The observed $\mathrm{m} / \mathrm{z} 319.2$ corresponding to Nile Red confirms the photoactivation product of SNile Red is its oxo form. The photo-oxidation quantum yield $\left(\Phi_{\mathrm{o}}\right)$ of SNile Red was assessed using LC-MS and revealed a moderate efficiency of $2.6 \%$ in DMSO under irradiation with $470 \mathrm{~nm}$ (470/60) light source.

Both dialkylthioketones and diarylthioketones are known to undergo a photo-oxidation reaction under oxygen and light to give the corresponding ketones. ${ }^{26,27}$ To investigate the effects of dissolved oxygen and light on the photoactivation of thio-caged fluorophores, the time profile of the fluorescence of SNile Red was measured in the presence or absence of light or oxygen. As shown in Figure 2E, the fluorescence intensity at $626 \mathrm{~nm}$ increased 90fold after irradiation with red light $(615 / 30 \mathrm{~nm})$ in the air for $20 \mathrm{~min}$, while no change regarding the fluorescent spectrum was observed in the absence of light. To study the effect of oxygen during the photoactivation, we prepared an oxygen-free SNile Red solution by bubbling nitrogen gas and irradiated at $615 \mathrm{~nm}$. Figure 2E shows that no significant change of fluorescence intensity was observed within $20 \mathrm{~min}$ irradiation without oxygen. 
Inspired by the photoactivation mechanism of thioketones, we hypothesized that singlet oxygen generated by self-sensitization could be the active species to oxidize thiocarbonyl groups within fluorophores. ${ }^{26,27}$ To test this hypothesis, 1,3-diphenylisobenzofuran (DPBF), a classical singlet oxygen detection reagent, was used to determine the involvement of singlet oxygen in these oxidative reactions. ${ }^{28,29}$ As shown in Figure $2 \mathrm{~F}$, the singlet oxygen generation was confirmed by the absorbance decrease of DPBF at $410 \mathrm{~nm}$ in methylene dichloride (DCM) during light irradiation $(615 / 30 \mathrm{~nm})$. By referring to methylene blue (MB) $\left(\Phi_{\Delta, \mathrm{MB}}=0.57\right.$ in dichloromethane $),{ }^{30}$ the singlet oxygen quantum yield $\left(\Phi_{\Delta}\right)$ of SNile Red was determined to be 0.36 after correcting the absorption over the $600-630 \mathrm{~nm}$ region. ${ }^{31,32}$ Furthermore, we carried out the SNile Red photoactivation experiments in the presence of sodium azide, a singlet oxygen quencher. ${ }^{33,34} \mathrm{We}$ found that the turn-on rate of SNile Red slowed down by half in the DMSO/PBS (pH 7.4) (v/v, 50/50) mixed solvent, compared to the nontreated group (Figure S2). Therefore, our data suggested that the photoactivation of SNile Red was likely mediated by singlet oxygen that was generated by thio-caged dye sensitization upon light irradiation.

\section{A New Class of Visible-Light-Activated Dyes.}

Encouraged by the excellent photoactivation properties of SNile Red, we used Lawesson's reagent to introduce thiocarbonyl moieties into several different fluorophores, such as phthalimide (PI), coumarin (Cou), and acridone (ACD), obtaining yields ranging from 20$80 \%$ (Figure 1). Next, we characterized the spectroscopic and photochemical properties of these thio-caged fluorophores and their photo-oxidation products using UV-vis and fluorescence spectroscopies (Table 1 and Figure S3). In general, the UV-vis absorption spectra of the thiocarbonyl fluorophores all exhibited distinct red-shifts compared to their carbonyl analogues. As shown in Table 1, thiocarbonyl group substitution led to significant bathochromic shifts in absorption peaks compared to the corresponding carbonyl compounds. Double thiocarbonyl substitution on 4-dimethylaminophthalimide (DMAP, Figure 1) produced an even larger bathochromic shift of $173 \mathrm{~nm}$. All the thiocarbonyl fluorophores exhibited larger extinction coefficients at their maximal absorption wavelengths than those of the corresponding carbonyl compounds. We hypothesized that the red-shift and enhanced absorption of thiocarbonyl compounds were due to the reduced energy gap between the HOMO and LUMO in these fluorophores. More importantly, thiocarbonyl substitutions in all tested fluorophores led to significant reductions in quantum yield, suggesting that replacement of a single oxygen atom with sulfur could be a general strategy for preparing quenched fluorophores with diverse structures. To assess the efficiency of photoactivation for the various thio-caged fluorophores, UV-vis and fluorescence spectra were recorded after different irradiation times (Figure S4-S8). All the thio-caged fluorophores underwent significant fluorescence enhancement after irradiation with light residing in their absorption wavelengths regime (Table 1). All the thiocaged dyes showed moderate photo-oxidation quantum yields from $1.2 \%-3.4 \%$ in DMSO (Table 1).

\section{Theoretical Study of Thio-Caged Fluorophores.}

To evaluate the mechanism responsible for fluorescence quenching in thiocarbonyl substituted fluorophores, we carried out density functional theory (DFT) calculations at the B3LYP/6-31G(d) level to reveal the energy levels of frontier molecular orbitals in thio- 
caged and uncaged fluorophores. Because of the availability of single crystals, we used ACD as a model. As shown in Figure S9, the HOMO-1 and LUMO of SACD are mainly constrained on the fluorophore phenyl moiety, while the HOMO of SACD is on the sulfur part. That is, for the whole molecule (SACD), the HOMO-1 and LUMO correspond to the HOMO and LUMO of the fluorophore core, respectively. After excitation, the electron in the HOMO-1 of SACD is promoted to the LUMO of the molecule. Because of the higher energy of the sulfur's HOMO ( $-5.61 \mathrm{eV}$, corresponding to HOMO in the whole molecule) than the fluorophore's HOMO $(-5.73 \mathrm{eV}$, corresponding to HOMO-1 in the whole molecule), the electron from the sulfur donor can transfer to the fluorophore, thus leading to the fluorescence quenching via PET mechanism. ${ }^{35}$ Upon the light irradiation, the desulfation reaction can reorganize the frontier molecular orbitals of the molecule. Both the LUMO and HOMO of ACD are localized on the fluorophore phenyl moiety. HOMO-1 $(-6.68 \mathrm{eV})$ is then constrained on the oxygen part, which is lower than the energy of the HOMO for the fluorophore $(-5.87 \mathrm{eV})$. Thus, the electron only can transfer between the HOMO and the LUMO of the fluorophore, which inhibits the PET process and restores the fluorescence.

\section{Fluorescence Imaging Using Thio-Caged Fluorophores.}

Before we tested the utility of thio-caged fluorophores for biological imaging, we evaluated the cytotoxicity and cellular permeability of these thio-caged fluorophores using A431 and 3T3-L1 cells, respectively. Cytotoxicity was evaluated by Cell Counting Kit-8. At a concentration of $4 \mu \mathrm{M}$ of the thio-caged fluorophores, $90 \%$ of A431 and 3T3-L1 cells remained viable even after $24 \mathrm{~h}$ (Figure S10). As shown in Figure 4A and Figure S11, after in situ irradiation of the cells, SACD, SDMAP, and SNile Red showed distinct intracellular fluorescence, suggesting their good cell permeability. The SCou did not show any intracellular fluorescence. We reasoned that the better reactivity of the thio-lactone motif may have introduced side-reactions upon culturing SCou with live cells, which prevented the formation of decaged coumarin fluorophore.

The stability of the SNile Red in solvents and cellular milieu was assessed. It showed more than $80 \%$ stability in PBS ( $\mathrm{pH} 7.4$ ) over $4 \mathrm{~h}$ based on the UV-vis absorption change (Figure S12). The intracellular stability of SNile Red in adipocytes was evaluated by confocal laser scanning microscope (CLSM). As shown in Figure S13, SNile Red pretreated adipocytes showed negligible fluorescence change during the following $1 \mathrm{~h}$ of incubation, indicating no undesired activation of SNile Red in the intracellular milieu. We then used thio-caged SNile Red to obtain subcellular structural data via fluorescence imaging of adipocytes. The specificity of SNile Red was determined by fluorescence localization experiments in adipocytes differentiated from 3T3-L1 cells using CLSM. The classical lipid droplet dye, BODIPY 493/503, was used as the marker. ${ }^{36}$ The adipocytes were stained with $2 \mu \mathrm{M}$ SNile Red for $10 \mathrm{~min}$ and then $100 \mathrm{nM}$ BODIPY 493/503 for $10 \mathrm{~min}$. As shown in Figure 3A, after irradiation with a $561 \mathrm{~nm}$ laser for $30 \mathrm{~s}$, the adipocytes displayed a globular red fluorescence from photo-activated SNile Red. The SNile Red fluorescence exhibited more than $90 \%$ colocalization with BODIPY 493/503 labeling, indicating that the two dyes were localized at the same cellular compartments. Our data, therefore, demonstrate that photoactivated SNile Red preserves the labeling selectivity of Nile Red and can serve as a lipiddroplet specific dye. 
To demonstrate the feasibility of spatial photoactivation of SNile Red, we used the $561 \mathrm{~nm}$ laser to irradiate several groups of adipocytes incubated with SNile Red in a sequential fashion, followed by fluorescence imaging. As shown in Figure S14, five groups of adipocytes lit up sequentially in the multicellular environment, indicating that the SNile Red photoactivatable probe holds the potential to track the dynamic events of lipid droplet behavior in a complex biological sample with excellent spatial resolution.

\section{Super-Resolution Imaging of Lipid Droplets Using Thio-Caged Fluorophores.}

Super-resolution imaging based on sequential imaging of a specific region of labeled tissue or cell culture relies on the ability to modulate the fluorescence of fluorophores between dark and bright states. ${ }^{10,11}$ As described above, SNile Red exhibits an excellent activation ratio of 280-fold, an attractive property for super-resolution imaging. Furthermore, the photoactivation of SNile Red can be achieved using visible light, affording lower phototoxicity and better tissue penetration than the use of UV light. To demonstrate the potential of thio-caged fluorophores for super-resolution imaging, we first used CLSM to investigate the processes of photoactivation and photobleaching for SNile Red in living adipocytes. A $561 \mathrm{~nm}$ laser was utilized to activate and bleach SNile Red. As shown in Figure 3B,C, and Video S1, differentiated 3T3-L1 adipocytes exhibited no significant fluorescence prior to photoactivation. Following irradiation with the $561 \mathrm{~nm}$ laser for $80 \mathrm{~s}$, a 5 -fold increase of fluorescence intensity was detected. The recovered intracellular fluorescence intensity of SNile Red is $80 \%$ of Nile Red at the identical concentration (Figure S15). Subsequently, photobleaching was performed in the same cells using the $561 \mathrm{~nm}$ laser. As shown in Figure 3B,C, and Video S2, after continuous scanning for $360 \mathrm{~s}$, the fluorescence intensity decayed to $10 \%$ of its maximum. This photoswitching flexibility holds promise for SNile Red utilization in further PALM studies.

Next, we explored the utility of SNile Red by performing super-resolution imaging of lipid droplets. Adipocytes differentiated from 3T3-L1 cells were stained with $2 \mu \mathrm{M}$ SNile Red and then washed prior to PALM imaging. During PALM imaging, SNile Red molecules went through photoactivation and photobleaching processes using simultaneous 405 and 561 $\mathrm{nm}$ laser stimulation. By capturing bright but sparse, stochastic events for a period of 20 000-30 000 imaging frames, we reconstructed the apparent surface of SNile Red-labeled lipid droplets with high molecular accuracy. Compared to the conventional widefield fluorescence image and differential interference contrast (DIC) image (Figure 4A), the PALM reconstruction outlines a pair of lipid droplets with a resolution beyond the optical diffraction limit (Figure 4A,B). The precision of localization, judged by error fitting of single molecules using the Thompson equation, ${ }^{37}$ was roughly 10-20 nm (Figure 4B). A line-scan through the larger lipid droplet (yellow dotted line) in Figure $4 \mathrm{~B}$ gave its diameter as $2.26 \mu \mathrm{m}$, which is within the referenced size of lipid droplets. ${ }^{38}$ To our knowledge, this is the first super-resolution imaging of lipid droplets in adipocytes using photoactivatable probes.

To explore the utility of thio-caged fluorophores for multicolor super-resolution imaging, we performed a two-color PALM imaging of SNile Red in early differentiated adipocytes in combination with Alexa Fluor 647-immunolabeled perilipin-1 (PLIN1). PLIN is a key 
regulator of lipolysis in adipocytes, which coats lipid droplet exclusively in adipocytes. ${ }^{39}$ As shown in Figure 4D and E, we successfully resolved the lipid droplet structure with localization accuracy smaller than $35 \mathrm{~nm}$ in both channels, where immunolabeled PLIN1 (red) located on the membrane structure and wrapped around the SNile Red-labeled lipid droplet hydrophobic core. Therefore, our thio-caged fluorophores showed good compatibility with other commercial dyes for biological imaging and are ready for multicolor imaging.

\section{Super-Resolution Imaging of HaloTag Proteins Using Thio-Caged Fluorophores.}

Encouraged by super-resolution studies of cellular organelles above, we explored the possibility of using these thio-caged fluorophores for protein imaging. To site-specifically label proteins of interest with thio-caged fluorophores, we used the HaloTag labeling technology that is widely applicable for live mammalian cells imaging. ${ }^{40}$ This technology is based on a spontaneous covalent bond formation between HaloTag-fused proteins and an exogenously added synthetic HaloTag ligand. Given the superb photoactivation and biochemistry property of SDMAP, we first prepared a HaloTag ligand modified by SDMAP (SDMAP-Halo in Figure 5A). Like the other thio-caged fluorophores, the resulting probe exhibited a 430-fold fluorescence enhancement upon irradiation with $365 \mathrm{~nm}$ hand-hold UV lamp (Figure S16). Next, CHO-K1 cells were transfected with a plasmid encoding histone 2B (H2B) fused with HaloTag, followed by the reaction with SDMAP-Halo. To our delight, we observed a distinct nuclear pattern labeling after photoactivation by $405 \mathrm{~nm}$ laser equipped in the CLSM, which is further confirmed by the colocalization imaging with the commercial nuclear dye, DRAQ5, (Figure 5C and Figure S17). The intracellular photoactivation process was recorded by Video S3, accompanied by a 43-fold fluorescence increase. The recovered intracellular fluorescence intensity of the SDMAP-Halo is $80 \%$ of DMAP-Halo at the identical concentration (Figure S18). Then, we performed superresolution imaging of histone-2B using this platform. As shown in Figure 5C, we could obtain PALM images of histones with a localization precision of ca. $38 \mathrm{~nm}$, suggesting the ability of the thio-caged fluorophores to obtain fine structures of target protein when combined with protein labeling technologies.

It is worth noting that our PALM imaging can be done in living cells under physiological conditions without adding cytotoxic redox chemicals or oxygen scavengers to induce the fluorescence on-off transition cycles. Taken together, the results of this study show that the thio-caged fluorophores developed are compatible with existing protein labeling technology, and can be used for living cell super-resolution imaging.

\section{CONCLUSION}

In summary, we have developed a general strategy for preparing photoactivatable probes by performing a single sulfur-for-oxygen atom substitution within fluorescent molecules. Using this strategy, a set of thio-caged fluorophores spanning a broad spectral range were synthesized and characterized. We found that these thio-caged fluorophores exhibited almost no fluorescence but could be readily converted to their strongly fluorescent oxidized analogues upon irradiation with low-power visible light in air. Calculations indicate that 
thiocarbonyl substitution in different fluorophores results in significant loss of the fluorescence signal via a photoinduced electron transfer quenching mechanism. More importantly, these thio-caged fluorophores can be photoactivated using light with similar wavelengths to their absorbance, rather than UV light with high phototoxicity and low tissue penetration. For example, thio-caged Nile Red (SNile Red, $\lambda_{\mathrm{Max}}=652 \mathrm{~nm}$ ) can be activated by $615 \mathrm{~nm}$ red light; thio-caged ACD (SACD, $\lambda_{\mathrm{Max}}=485 \mathrm{~nm}$ ) can be photoactivated using $470 \mathrm{~nm}$ blue light. We have demonstrated the utility of thio-caged fluorophores for multicolor imaging by obtaining super-resolution images of lipid droplets with a localization precision of ca. $13 \mathrm{~nm}$. In addition, these thio-caged fluorophores can serve as the substrates for protein tag to site-specifically label the proteins of interest, which is applicable to the living cell PALM imaging with low-power activation light under physiological conditions in the absence of cytotoxic additives. Compared to widely used $o$-nitrobenzyl photoactivatable dyes, these thio-caged fluorophores have several significant advantages, including ease of design and synthesis, requirement for minimal molecular modification and high efficiency of unmasking using visible light. Furthermore, it is noteworthy that these thio-caged fluorophores only harbor one atom change compared to the parent fluorophores, which is likely to minimize the perturbations to biological systems. Given the versatility of this strategy for designing photo-activatable dyes, we envision that these thio-caged fluorophores can be used as diverse probes for exploring a wide variety of biological processes.

\section{Supplementary Material}

Refer to Web version on PubMed Central for supplementary material.

\section{ACKNOWLEDGMENTS}

This work was supported by the Cancer Prevention Research Institute of Texas (CPRIT, RR170014), the Robert A. Welch Foundation (C-1970, Q-0035, and BE-1913), the Hamill Innovation Award (Hamill Foundation), the John S. Dunn Foundation Collaborative Research Award (Gulf Coast Consortia) and NIH (R35-GM133706, F32EY027171, R01-GM112003, and R01-EY026545).

\section{REFERENCES}

(1). Lavis LD; Chao T-Y; Raines RT Fluorogenic Label for Biomolecular Imaging. ACS Chem. Biol 2006, 1 (4), 252-260. [PubMed: 17163679]

(2). Puliti D; Warther D; Orange C; Specht A; Goeldner M Small Photoactivatable Molecules for Controlled Fluorescence Activation in Living Cells. Bioorg. Med. Chem 2011, 19 (3), 1023 1029. [PubMed: 20675143]

(3). Brieke C; Rohrbach F; Gottschalk A; Mayer G; Heckel A Light-Controlled Tools. Angew. Chem., Int. Ed 2012, 51 (34), 8446-8476.

(4). Li W; Zheng G Photoactivatable Fluorophores and Techniques for Biological Imaging Applications. Photochemical \& Photobiological Sciences 2012, 11 (3), 460. [PubMed: 22252510]

(5). Grimm JB; Heckman LM; Lavis LD Chapter One-The Chemistry of Small-Molecule Fluorogenic Probes. In Progress in Molecular Biology and Translational Science; Morris MC, Ed.;Fluorescence-Based Biosensors; Academic Press, 2013; Vol. 113, pp 1-34. DOI: 10.1016/ B978-0-12-386932-6.00001-6.

(6). Chozinski TJ; Gagnon LA; Vaughan JC Twinkle, Twinkle Little Star: Photoswitchable Fluorophores for Super-Resolution Imaging. FEBS Lett. 2014, 588 (19), 3603-3612. [PubMed: 25010263] 
(7). Grimm JB; English BP; Choi H; Muthusamy AK; Mehl BP; Dong P; Brown TA; LippincottSchwartz J; Liu Z; Lionnet T; et al. Bright Photoactivatable Fluorophores for Single-Molecule Imaging. Nat. Methods 2016, 13 (12), 985-988. [PubMed: 27776112]

(8). Wijesooriya CS; Peterson JA; Shrestha P; Gehrmann EJ; Winter AH; Smith EA A Photoactivatable BODIPY Probe for Localization-Based Super-Resolution Cellular Imaging. Angew. Chem., Int. Ed 2018, 57 (39), 12685-12689.

(9). Zhang Y; Song K-H; Tang S; Ravelo L; Cusido J; Sun C; Zhang HF; Raymo FM Far-Red Photoactivatable BODIPYs for the Super-Resolution Imaging of Live Cells. J. Am. Chem. Soc 2018, 140 (40), 12741-12745. [PubMed: 30247890]

(10). Bates M; Huang B; Dempsey GT; Zhuang X Multicolor Super-Resolution Imaging with PhotoSwitchable Fluorescent Probes. Science 2007, 317 (5845), 1749-1753. [PubMed: 17702910]

(11). Sengupta P; van Engelenburg SB; Lippincott-Schwartz J Superresolution Imaging of Biological Systems Using Photoactivated Localization Microscopy. Chem. Rev 2014, 114 (6), 3189-3202. [PubMed: 24417572]

(12). Nani RR; Gorka AP; Nagaya T; Kobayashi H; Schnermann MJ Near-IR Light-Mediated Cleavage of Antibody-Drug Conjugates Using Cyanine Photocages. Angew. Chem 2015, 127 (46), 13839-13842.

(13). Atilgan A; Tanriverdi Ecik E; Guliyev R; Uyar TB; Erbas-Cakmak S; Akkaya EU Near-IRTriggered, Remote-Controlled Release of Metal Ions: A Novel Strategy for Caged Ions. Angew. Chem., Int. Ed 2014, 53 (40), 10678-10681.

(14). Brown EB; Shear JB; Adams SR; Tsien RY; Webb WW Photolysis of Caged Calcium in Femtoliter Volumes Using Two-Photon Excitation. Biophys. J 1999, 76 (1), 489-499. [PubMed: 9876162]

(15). Tran C; Gallavardin T; Petit M; Slimi R; Dhimane H; Blanchard-Desce M; Acher FC; Ogden D; Dalko PI Two-Photon "Caging" Groups: Effect of Position Isomery on the Photorelease Properties of Aminoquinoline-Derived Photolabile Protecting Groups. Org. Lett 2015, 17 (3), 402-405. [PubMed: 25625881]

(16). Goswami PP; Syed A; Beck CL; Albright TR; Mahoney KM; Unash R; Smith EA; Winter AH BODIPY-Derived Photoremovable Protecting Groups Unmasked with Green Light. J. Am. Chem. Soc 2015, 137 (11), 3783-3786. [PubMed: 25751156]

(17). Rubinstein N; Liu P; Miller EW; Weinstain R Meso-Methylhydroxy BODIPY: A Scaffold for Photo-Labile Protecting Groups. Chem. Commun 2015, 51 (29), 6369-6372.

(18). Peterson JA; Wijesooriya C; Gehrmann EJ; Mahoney KM; Goswami PP; Albright TR; Syed A; Dutton AS; Smith EA; Winter AH Family of BODIPY Photocages Cleaved by Single Photons of Visible/Near-Infrared Light. J. Am. Chem. Soc 2018, 140 (23), 7343-7346. [PubMed: 29775298]

(19). Goldberg JM; Batjargal S; Petersson EJ Thioamides as Fluorescence Quenching Probes: Minimalist Chromophores to Monitor Protein Dynamics. J. Am. Chem. Soc 2010, 132 (42), 14718-14720. [PubMed: 20886849]

(20). Goldberg JM; Speight LC; Fegley MW; Petersson EJ Minimalist Probes for Studying Protein Dynamics: Thioamide Quenching of Selectively Excitable Fluorescent Amino Acids. J. Am. Chem. Soc 2012, 134 (14), 6088-6091. [PubMed: 22471784]

(21). Goldberg JM; Batjargal S; Chen BS; Petersson EJ Thioamide Quenching of Fluorescent Probes through Photoinduced Electron Transfer: Mechanistic Studies and Applications. J. Am. Chem. Soc 2013, 135 (49), 18651-18658. [PubMed: 24266520]

(22). Huang Y; Ferrie JJ; Chen X; Zhang Y; Szantai-Kis DM; Chenoweth DM; Petersson EJ Electronic Interactions of i, i + 1 Dithioamides: Increased Fluorescence Quenching and Evidence for n-to$\Pi *$ Interactions. Chem. Commun 2016, 52 (50), 7798-7801.

(23). Choi MG; Kim YH; Namgoong JE; Chang S-K Hg2+-Selective Chromogenic and Fluorogenic Chemodosimeter Based on Thiocoumarins. Chem. Commun. (Cambridge, U. K.) 2009, No. 24, 3560-3562.

(24). Moon JO; Lee JW; Choi MG; Ahn S; Chang S-K Dual Signaling of Hypochlorous Acid by Desulfurization of Thiocoumarin. Tetrahedron Lett. 2012, 53 (48), 6594-6597.

(25). Park JE; Choi MG; Chang S-K Colorimetric and Fluorescent Signaling of Au3+ by Desulfurization of Thiocoumarin. Inorg. Chem 2012, 51 (5), 2880-2884. [PubMed: 22324661] 
(26). Coyle JD The Photochemistry of Thiocarbonyl Compounds. Tetrahedron 1985, 41 (23), 53935425.

(27). Corsaro A; Pistarà V Conversion of the Thiocarbonyl Group into the Carbonyl Group. Tetrahedron 1998, 54 (50), 15027-15062.

(28). Spiller W; Kliesch H; Wöhrle D; Hackbarth S; Röder B; Schnurpfeil G Singlet Oxygen Quantum Yields of Different Photosensitizers in Polar Solvents and Micellar Solutions. J. Porphyrins Phthalocyanines 1998, 2 (2), 145-158.

(29). Tang J; Chen J-J; Jing J; Chen J-Z; Lv H; Yu Y; Xu P; Zhang J-L $\beta$-Lactonization of Fluorinated Porphyrin Enhances LDL Binding Affinity, Cellular Uptake with Selective Intracellular Localization. Chem. Sci 2014, 5 (2), 558-566.

(30). Li W; Li L; Xiao H; Qi R; Huang Y; Xie Z; Jing X; Zhang H Iodo-BODIPY: A Visible-LightDriven, Highly Efficient and Photostable Metal-Free Organic Photocatalyst. RSC Adv. 2013, 3 (32), 13417-13421.

(31). Bonacin JA; Engelmann FM; Severino D; Toma HE; Baptista MS Singlet Oxygen Quantum Yields (Фd) in Water Using Beetroot Extract and an Array of LEDs. J. Braz. Chem. Soc 2009, 20 (1), 31-36.

(32). Adarsh N; Avirah RR; Ramaiah D Tuning Photosensitized Singlet Oxygen Generation Efficiency of Novel Aza-BODIPY Dyes. Org. Lett 2010, 12 (24), 5720-5723. [PubMed: 21090576]

(33). Li MY; Cline CS; Koker EB; Carmichael HH; Chignell CF; Bilski P Quenching of Singlet Molecular Oxygen (1O2) by Azide Anion in Solvent Mixtures. Photochem. Photobiol 2001, 74 (6), 760-764. [PubMed: 11783930]

(34). Bancirova M Sodium Azide as a Specific Quencher of Singlet Oxygen during Chemiluminescent Detection by Luminol and Cypridina Luciferin Analogues. Luminescence 2011, 26 (6), 685-688. [PubMed: 21491580]

(35). Escudero D Revising Intramolecular Photoinduced Electron Transfer (PET) from First-Principles. Acc. Chem. Res 2016, 49 (9), 1816-1824. [PubMed: 27575871]

(36). Fam TK; Klymchenko AS; Collot M Recent Advances in Fluorescent Probes for Lipid Droplets. Materials 2018, 11 (9), 1768.

(37). Thompson RE; Larson DR; Webb WW Precise Nanometer Localization Analysis for Individual Fluorescent Probes. Biophys. J 2002, 82 (5), 2775-2783. [PubMed: 11964263]

(38). Guo Y; Cordes KR; Farese RV; Walther TC Lipid Droplets at a Glance. J. Cell Sci 2009, 122 (6), 749-752. [PubMed: 19261844]

(39). Brasaemle DL Thematic Review Series: Adipocyte Biology. The Perilipin Family of Structural Lipid Droplet Proteins: Stabilization of Lipid Droplets and Control of Lipolysis. J. Lipid Res 2007, 48 (12), 2547-2559. [PubMed: 17878492]

(40). England CG; Luo H; Cai W HaloTag Technology: A Versatile Platform for Biomedical Applications. Bioconjugate Chem. 2015, 26 (6), 975-986. 

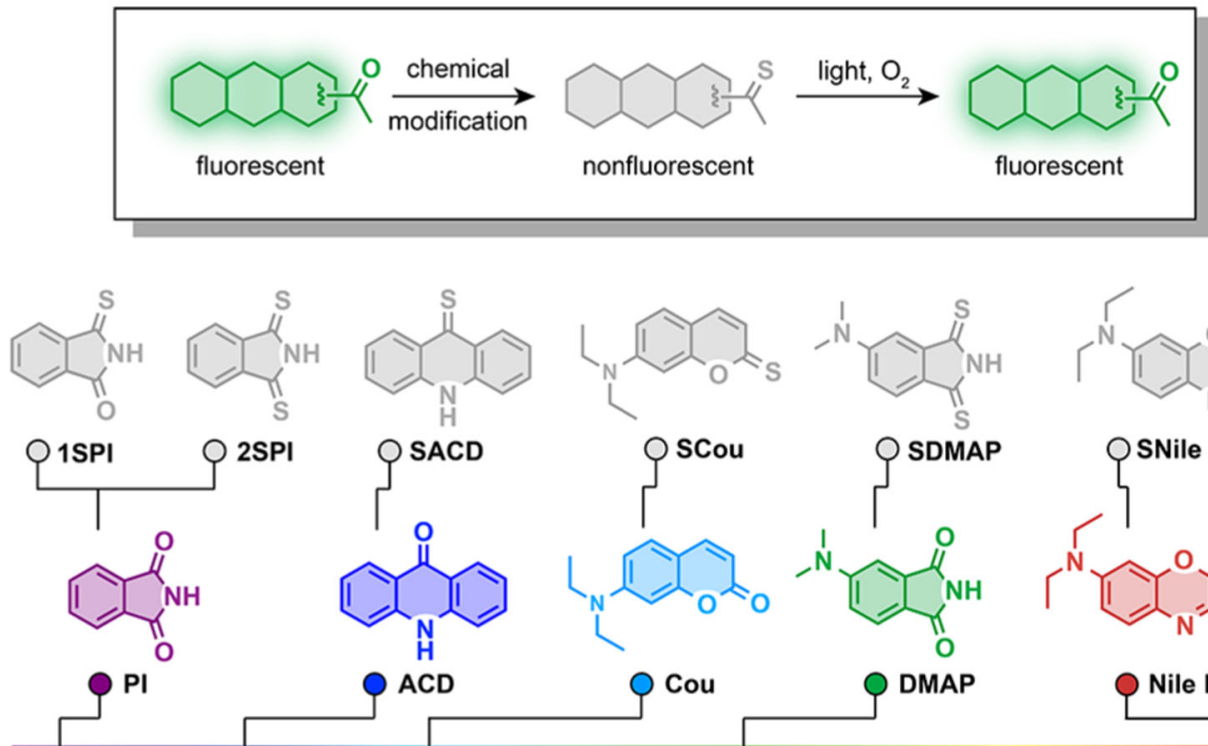

$350 \mathrm{~nm}$

Figure 1.

Design of fluorogenic dyes (top). Thiocarbonyl substitution at the carbonyl group of fluorophores results in very weak fluorescence via a PET-quenching mechanism. Upon irradiation with light, the thiocarbonyl group can be efficiently desulfurized to its oxo derivative, thus restoring strong fluorescence of the fluorophores. Structures of thio-caged and uncaged fluorophores described in this study (bottom). 
A
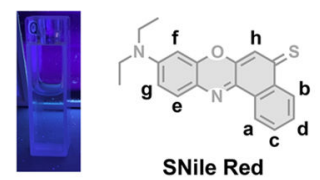

$\stackrel{\text { Red light }}{\longrightarrow}$
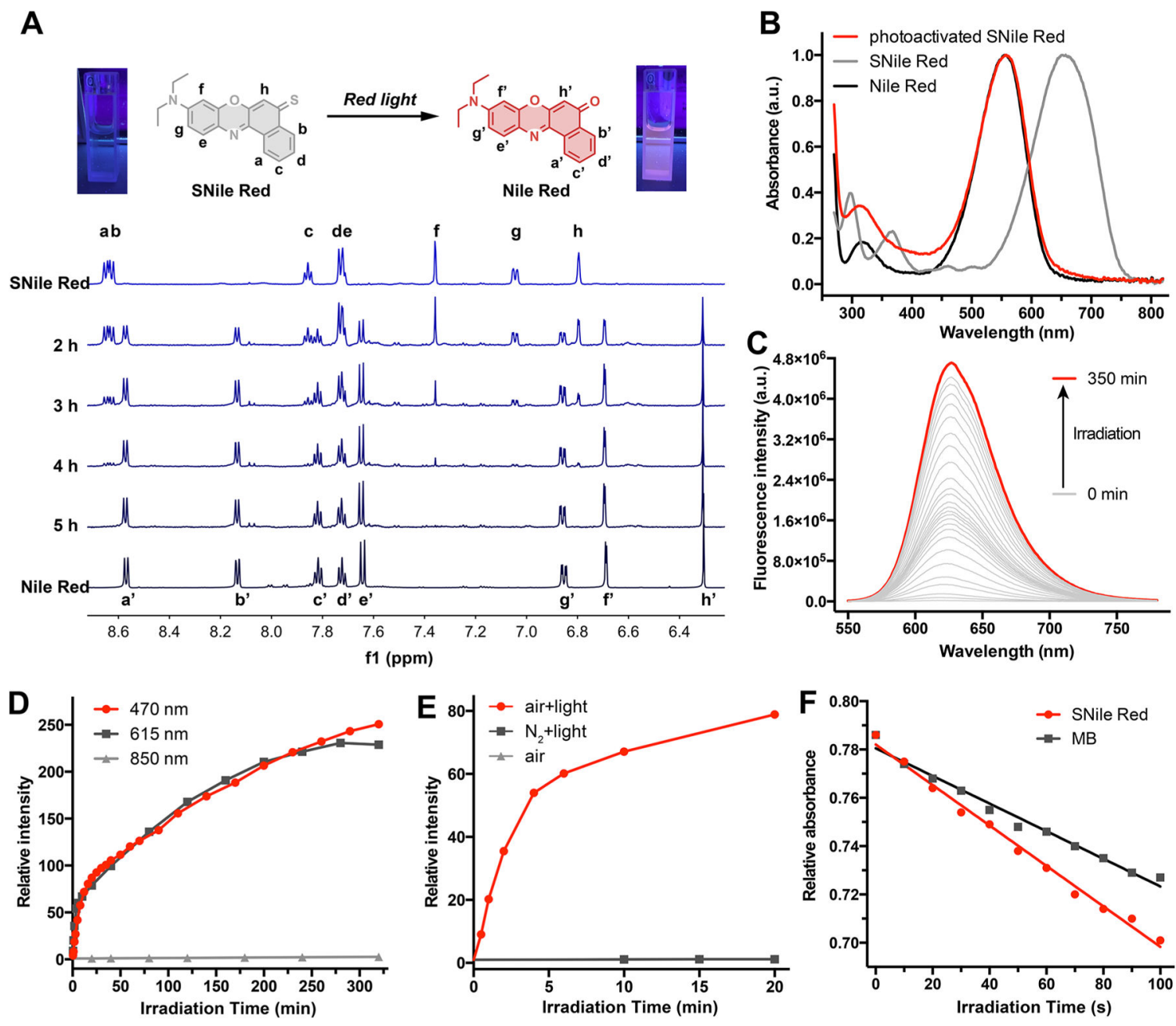

Figure 2.

(A) Overlay of ${ }^{1} \mathrm{HNMR}$ spectra (6.2-8.8 ppm) of SNile Red taken at the indicated light irradiation times ( $\left.615 \mathrm{~nm}, 0.4 \mu \mathrm{W} \mathrm{cm}{ }^{-2}\right)$. (B) Normalized absorbance spectra of Nile Red, SNile Red, and SNile Red after photoactivation. (C) Fluorescence spectra of SNile Red after light irradiation for different times ( $470 \mathrm{~nm}, 0.4 \mu \mathrm{W} \mathrm{cm}^{-2}$ ). (D) Fluorescence change of SNile Red irradiated with different wavelengths of light ( $470 \mathrm{~nm}, 0.4 \mu \mathrm{W} \mathrm{cm}{ }^{-2} ; 615 \mathrm{~nm}, 0.4$ $\mu \mathrm{W} \mathrm{cm}{ }^{-2} ; 850 \mathrm{~nm}, 0.4 \mu \mathrm{W} \mathrm{cm}^{-2}$ ). (E) Fluorescence change of SNile Red in the presence or absence of light, or oxygen. (F) Absorbance change of DPBF at $410 \mathrm{~nm}$ in the presence of SNile Red or methylene blue (MB) in DCM after different irradiation times. 


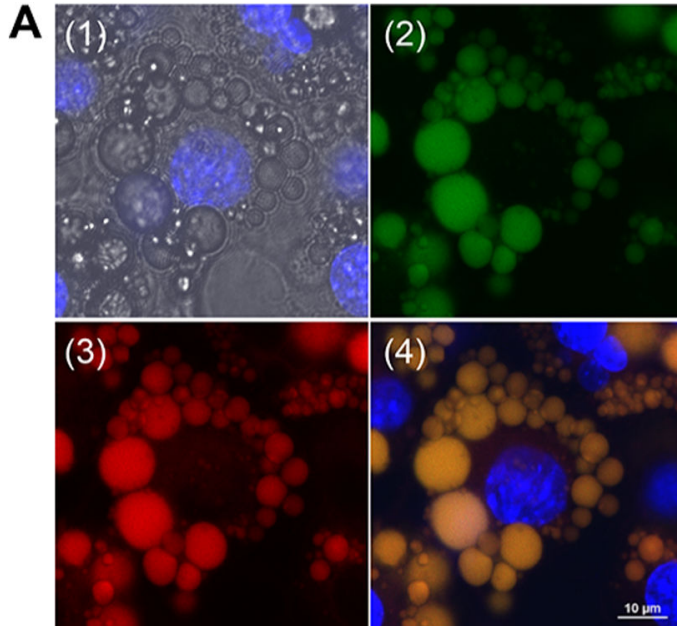

B

C

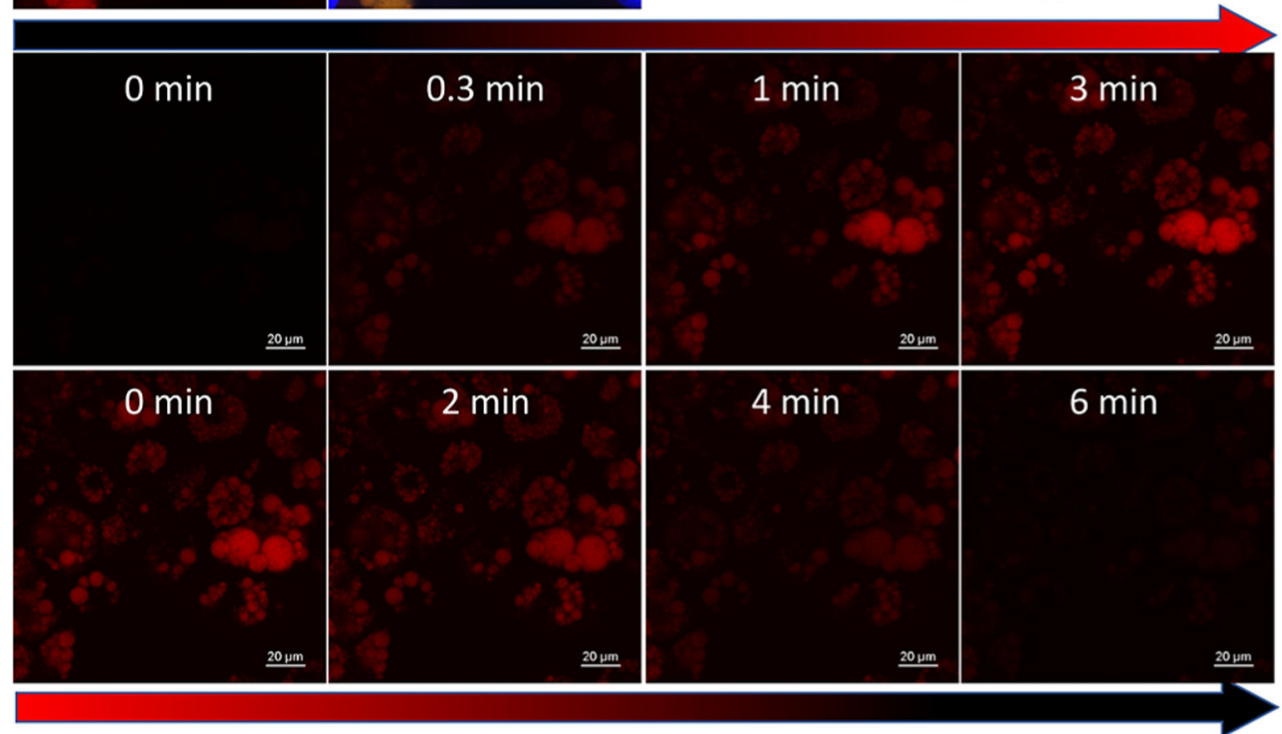

Figure 3.

(A) Signals from photoactivated SNile Red colocalize with BODIPY 493/503. Adipocytes were incubated with SNile Red, BODIPY 493/503, and Hoechst 33342, followed by photoactivation using the $561 \mathrm{~nm}$ laser. Scale bar: $10 \mu \mathrm{m}$. (B) A plot of the changes in relative intracellular fluorescence intensity of SNile Red during photoactivation and photobleaching in living adipocytes using a $561 \mathrm{~nm}$ laser, respectively. (C) Confocal fluorescence images of living adipocytes using SNile Red during photoactivation and photobleaching. Scale bar: $20 \mu \mathrm{m}$. 


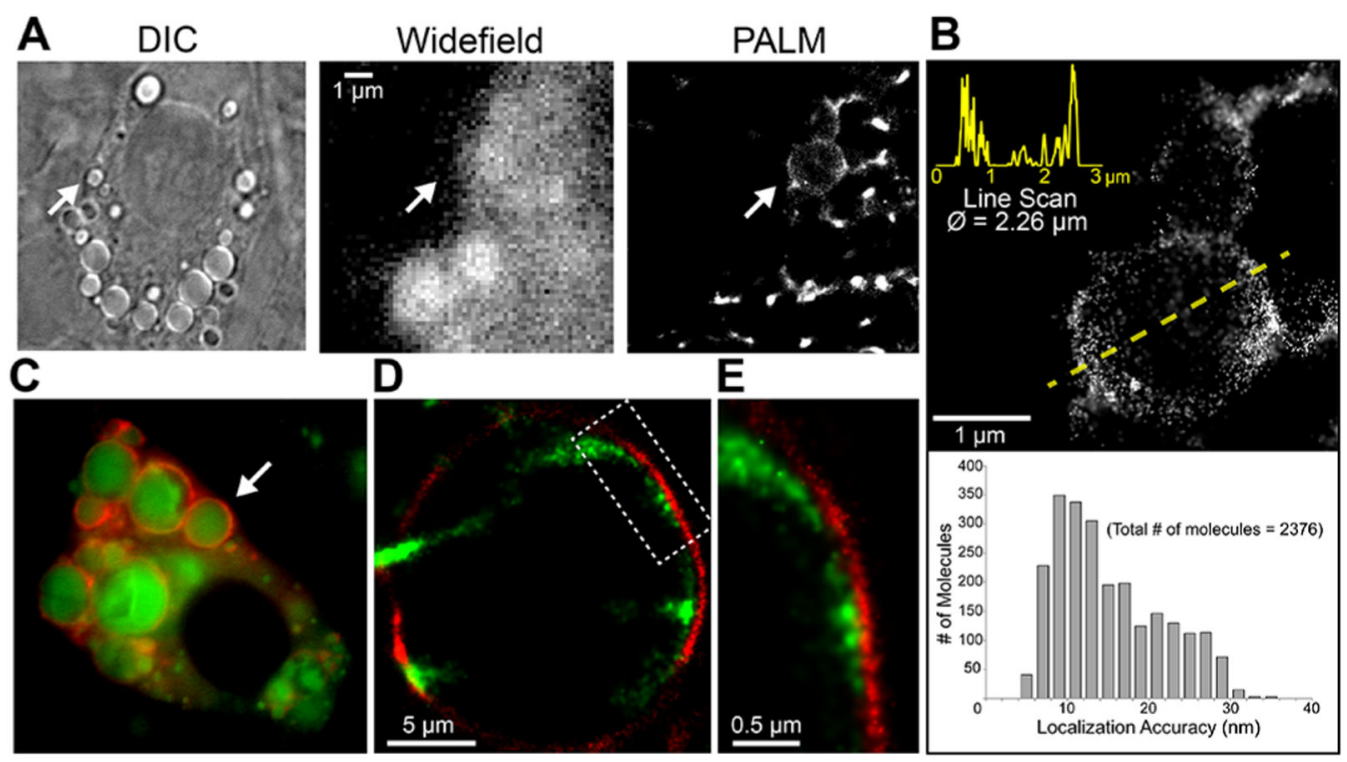

Figure 4.

(A) Differential interference contrast (DIC), widefield, and PALM images of an adipocyte labeled with SNile Red (2 $\mu \mathrm{M})$. (B) Magnified region featuring SNile Red labeled lipid droplets by super-resolution reconstruction via PALM localization of spontaneous reactivation fluorescent events and corresponding histogram plot of the localization accuracy of reconstructed molecules. A line-scan through the larger droplet (yellow dotted line) was used to plot the diameter $(\varnothing)$. (C) Two-color widefield fluorescence image of an adipocyte. Red and green colors indicated Alexa Fluor 647 labeled PLIN1 and SNile Red, respectively. (D) Corresponding two-color super-resolution reconstruction of the lipid droplet indicated by the white arrow in panel $\mathrm{C}$, and $(\mathrm{E})$ a magnified view. 

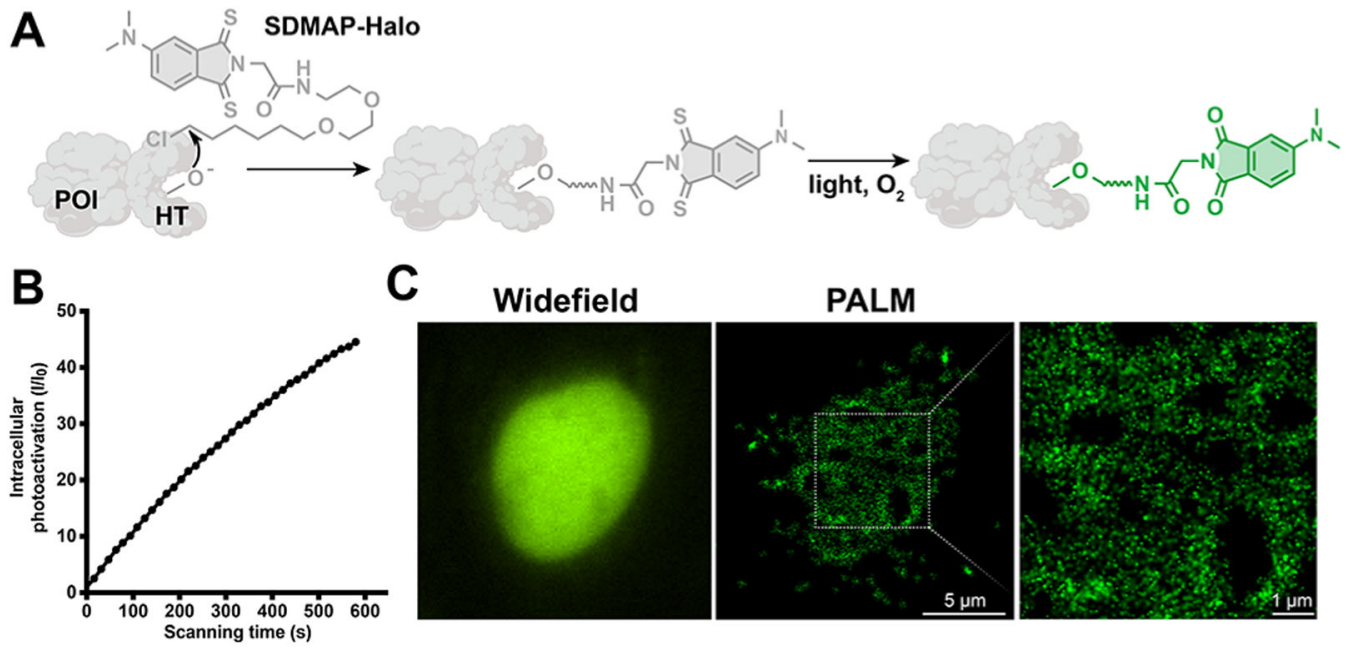

Figure 5.

(A) Scheme of the labeling of the protein of interest (POI) with the SDMAP-Halo ligand for fluorescence imaging. (B) A plot of the changes in relative intracellular fluorescence intensity of SDMAP-Halo during photoactivation in living CHO-K1 cells using a $405 \mathrm{~nm}$ laser. (C) A widefield fluorescence image and corresponding PALM reconstruction of SDMAP-Halo nuclear labeling in a H2B-HaloTag expressing CHO-K1 cell. 


\section{올

I



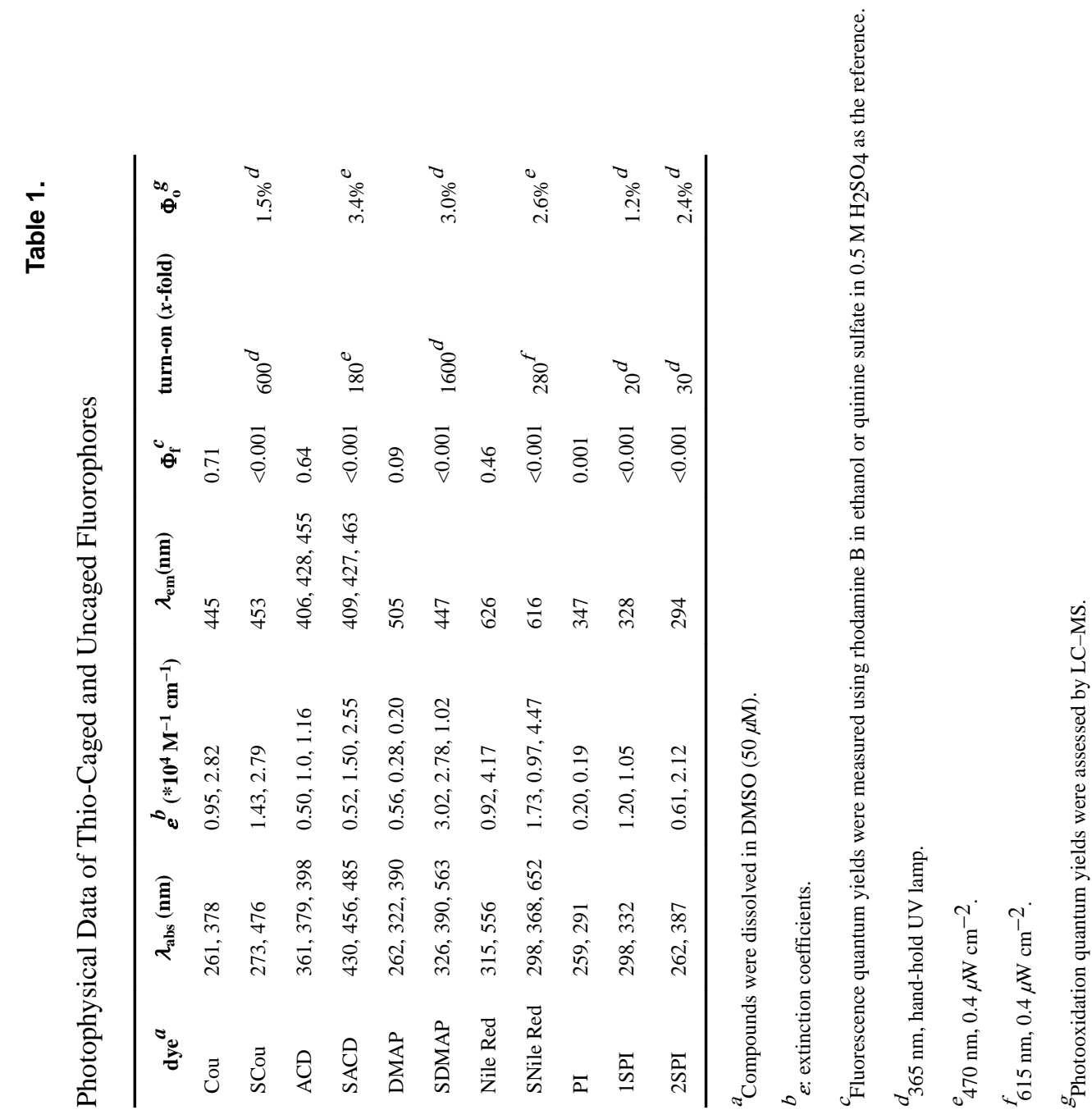

J Am Chem Soc. Author manuscript; available in PMC 2019 October 24. 